Bundesgesundheitsbl 2016 $\cdot 59: 292-299$

DOI 10.1007/s00103-015-2290-7

Online publiziert: 2. Dezember 2015

(c) Springer-Verlag Berlin Heidelberg 2015

CrossMark
Nadja Neumann ${ }^{1} \cdot$ Dorothea Mischler $^{1} \cdot$ Christiane Cuny $^{2} \cdot$ Michael Hogardt $^{3}$. Volkhard A.J. Kempf ${ }^{3}$. Ursel Heudorf ${ }^{1}$

${ }^{1}$ Gesundheitsamt Frankfurt, MRE-Netz Rhein-Main, Frankfurt am Main, Deutschland

2 Nationales Referenzzentrum für Staphylokokken, Wernigerode, Deutschland

${ }^{3}$ Institut für medizinische Mikrobiologie und Krankenhaushygiene,

Universitätsklinikum Frankfurt am Main, Frankfurt am Main, Deutschland

\section{Multiresistente Erreger bei Patienten ambulanter Pflegedienste im Rhein-Main-Gebiet 2014}

\section{Prävalenz und Risikofaktoren}

Multiresistente Erreger (MRE) sind ein großes Problem im Gesundheitswesen. Laut aktuellem Bericht der Europäischen Gesundheitsbehörde nimmt die MRSAProblematik (methicillinresistenter Staphylococcus aureus) in vielen Ländern Europas ab, wohingegen in praktisch allen Ländern eine Zunahme der Antibiotikaresistenzen bei den gramnegativen Erregern zu beobachten ist. Insbesondere gramnegative Erreger mit Resistenzen gegen drei Antibiotikagruppen (3MRGN) und solche mit Resistenzen gegen vier Antibiotikagruppen, also einschließlich Resistenz gegen Carbapeneme (4MRGN), nehmen in klinischen Isolaten deutlich zu $[1,2]$.

Die meisten MRSA-Stämme sind mit medizinischen Einrichtungen und Behandlungen assoziiert (sog. haMRSA, healthcare associated MRSA), die MRSA-Prävalenz in der Allgemeinbevölkerung in Deutschland liegt bei $0,5 \%$ [3]. Demgegenüber konnten verschiedene Studien zeigen, dass gramnegative Erreger, insbesondere Enterobakterien mit erweiterter Resistenz gegen $\beta$-Laktamantibiotika (ESBL), oft auf Auslandsreisen erworben und als „stumme Besiedler" unerkannt nach Hause mitgebracht werden können [4-6]. Die Prävalenz von Escherichia coli mit erweiterter Resistenz gegen $\beta$-Laktamantibiotika in der Allgemeinbevölkerung in Deutschland lag in einer in den Jahren 2009 bis 2012 in Bayern durchgeführten Studie bei $6,3 \%$, wobei $10 \%$ dieser Stämme auch eine Resistenz gegen Fluorchinolone aufwiesen, also als 3MRGN einzustufen waren [7].

Zwar wurden einige wenige Untersuchungen zur MRSA-Prävalenz bei Patienten ambulanter Pflegedienste publiziert [8-10], doch gibt es bislang keine Untersuchung zur Prävalenz multiresistenter gramnegativer Erreger (MRGN) in der ambulanten Pflege. Vor diesem Hintergrund wurde im MRENetz Rhein-Main eine Untersuchung zur MRE-Problematik bei Patienten ambulanter Pflegedienste vorgenommen, mit dem Ziel, nicht nur aktuelle Prävalenzdaten, sondern auch Hinweise auf etwaige Risikofaktoren für MRGN zu erhalten.

\section{Material und Methoden}

Vierzig der ca. 400 ambulanten Pflegedienste in der Rhein-Main-Region sind Mitglied im MRE-Netz Rhein-Main. Alle 40 am Netzwerk teilnehmenden Pflegedienste wurden über die geplante Untersuchung informiert mit der Bitte, daran teilzunehmen. Zehn Pflegedienste (25\% der Mitglieder, ca. 2,5\% aller Pflege- dienste) erklärten sich zur Teilnahme bereit, klärten ihre Patienten über das Untersuchungsziel auf und baten sie um das schriftliche Einverständnis zur Teilnahme. Für die Anamnesen wurde der HALT-Fragebogen leicht modifiziert übernommen. Dieser Fragebogen ist ein etabliertes Erhebungsinstrument zur europaweiten Untersuchung von Infektionen und des Antibiotikaeinsatzes in Altenpflegeheimen (HALT: healthcare associated infections in long-term care facilities) [11, 12]. Die Anamnesen und Abstrichuntersuchungen wurden von Oktober bis Dezember 2014 durchgeführt. Die Nasen-, Rachen- und die Analabstriche wurden mit Abstrichröhrchen der Fa. nerbe plus genommen und im Institut für Medizinische Mikrobiologie und Krankenhaushygiene des Universitätsklinikums Frankfurt untersucht.

Zum Nachweis von MRSA wurden die entnommenen Tupfer auf einem chromogenen Selektivmedium (Brilliance MRSA 2 Agar, Fa. Oxoid, Wesel, Deutschland) angelegt. Die Untersuchung auf ESBL resp. MRGN erfolgte mittels ESBLScreening-Agar (Chromagar ESBL, Fa. MAST Diagnostika, Reinfeld, Deutschland). Die Speziesdifferenzierung verdächtiger Kolonien erfolgte entsprechend den Herstellerangaben jeweils nach 24-stündiger Bebrütung bei $37^{\circ} \mathrm{C}$ 
mittels MALDI-TOF (VITEK-MS, Fa. bioMérieux, Nürtingen, Deutschland), die Resistenztestung von Reinkulturen u. a. mittels VITEK-2 (Fa. bioMérieux, Nürtingen, Deutschland). Weitere Untersuchungen beinhalteten kulturbasierte Identifikations- und Resistenzbestimmungsverfahren (z. B. modifizierter Hodge-Test) sowie diverse molekulare Typisierungs- und Identifikationsverfahren. Alle Untersuchungen liefen unter akkreditierten Bedingungen ab (DIN ISO 15189:2007; Urkunde D-ML-13102-1 und DIN ISO 17025:2005; Urkunde DPL-13102-1). Als ESBL wurden in dieser Arbeit Erreger mit erweiterter Resistenz gegen $\beta$-Laktamantibiotika eingestuft, wenn keine zusätzliche Resistenz gegen Fluorchinolone und/oder Carbapeneme vorlag. Bei 3MRGN und 4MRGN wurden die Definitionen der KRINKO [13] zugrunde gelegt, d. h. Resistenz gegen Leitantibiotika von drei resp. vier Antibiotikagruppen; 3MRGN: Resistenz gegen Piperacillin/Tazobactam, Cefotaxim und/ oder Ceftazidim, Ciprofloxacin; 4 MRGN: zusätzliche Resistenz gegen Imipenem und/oder Meropenem.

Jeder Patient erhielt vom Pflegedienst eine Pseudonymisierungsnummer, die auch auf dem Anamnesebogen und den mikrobiologischen Proben vermerkt war. Das MRE-Netz und das Labor erhielten nur Kenntnis dieser Nummer, personenbezogene Daten wie Namen verblieben in der Einrichtung. Die mikrobiologischen Ergebnisse wurden mit dieser Pseudonymisierungsnummer wieder an die Einrichtung zurückgespiegelt, die mit einer Decodierliste die individuelle Personenzuordnung wieder herstellen konnte. Bei MRSA-Nachweis bestand die Möglichkeit, vom Netzwerk kostenlos ein Sanierungskit anzufordern.

Die Auswertung der Daten erfolgte mit dem Statistikprogramm SPSS, Version 15. Berechnet wurden prozentuale Häufigkeiten, Gruppenunterschiede zwischen dem Intensivpflegedienst und den anderen Pflegediensten mit dem MannWhitney-Test und die Risikofaktoren der Besiedelung mit MRSA, ESBL/MRGN mittels Odds Ratio und logistischer Regression.

\section{Ergebnisse}

Von insgesamt 486 Patienten wurden die anamnestischen Daten erhoben, 269 (55,3\%) von ihnen ließen einen Rachenund/oder Nasenabstrich auf MRSA entnehmen, 132 (27,3\%) stimmten einem Analabstrich zu. Die Daten zu den Patientencharakteristika einschließlich der Risikofaktoren für MRE, die Angaben zu Krankenhausaufenthalten, Antibiotikatherapien, Operationen und der Besiedelung oder Infektion mit multiresistenten Erregern in der Anamnese sowie die Ergebnisse der mikrobiologischen Untersuchungen der entnommenen Abstriche sind in $\bullet$ Tab. 1 zusammengefasst. Jeweils ein Drittel der Patienten war männlich und/oder älter als 85 Jahre, $13 \%$ waren in Pflegestufe 3 oder höher eingestuft. Jeder fünfte Patient litt an Diabetes mellitus oder war desorientiert, knapp $40 \%$ waren inkontinent (jeweils zur Hälfte stuhl- oder harninkontinent). Hautbarriereverletzungen (Dekubitus oder andere Wunden) wurden bei $10 \%$ der Patienten angegeben, Harnwegskatheter bei 8,4\% und Gefäßkatheter bei $0,6 \%$. Knapp 5\% der Patienten waren mit einer perkutanen gastroskopischen Gastrostomie (PEG), $3 \%$ mit einem Tracheostoma, 1,6\% mit einem Kolostoma und 0,8\% mit einem Urostoma versorgt. Bettlägerigkeit lag bei $6 \%$ und eine Bewegungseinschränkung mit Rollstuhlpflicht bei $17 \%$ der Patienten vor. Von den Patienten hatten $11 \%$ in den letzten drei Monaten Antibiotika erhalten, 23\% waren innerhalb der vorangegangenen sechs Monate im Krankenhaus stationär behandelt worden und 2,5\% hatten sich innerhalb der letzten 30 Tage einer Operation unterziehen müssen.

Anamnestisch wurden bei über 2,5\% der Patienten eine MRSA-Besiedelung in der Vorgeschichte berichtet, bei $1,2 \%$ wurde noch aktuell eine MRSABesiedelung angegeben. ESBL/MRGN in der Vorgeschichte wurde bei 2,1\% der Patienten und eine aktuelle ESBLBesiedelung bei 0,6\% der Patienten angegeben.

Am Tag der Abstrichentnahme erhielten insgesamt fünf Patienten (1\%) ein Antibiotikum $(2 \times$ Fluorchinolone, $1 \times$ Cotrimoxazol, $1 \times$ Colistin, $1 \times$ keine genaue Angabe), bei 5 (1\%) Patienten wurde eine Infektion angegeben $(3 \times$ Harnwegsinfektion, $1 \times$ Atemwegsinfektion, $1 \times$ Haut-/Wundinfektion). Diese im Vergleich mit der Situation in Krankenhäusern niedrige Prävalenz an Antibiotikatherapien könnte ein Hinweis darauf sein, dass der Selektionsdruck für die Entstehung von Resistenzen gegen Antibiotika im Bereich der ambulanten Pflege deutlich niedriger ist. Auch die Gefahr der Erregerübertragung auf andere Patienten ist in der ambulanten Pflege geringer als im Krankenhaus oder der stationären Pflege, da eine Erregerübertragung ausschließlich (bei nicht sachgerechter Hygiene) über das Personal möglich ist, während im stationären Setting auch direkte Übertragungen von Patient zu Patient resp. von Bewohner zu Bewohner oder auch eine indirekte Übertragung über (nicht sachgerecht aufbereitete) Flächen möglich ist.

Die Charakteristika der Patienten des Intensivpflegedienstes unterschieden sich deutlich von denen der anderen Pflegedienste ohne Intensivpflege (s. - Tab. 1). Keiner der Patienten des Intensivpflegedienstes war älter als 85 Jahre (andere Pflegedienste: $37,8 \%$ ). Im Vergleich mit den Patienten anderer Pflegedienste waren die Patienten des Intensivpflegedienstes ( $n=20)$ häufiger männlich (50 vs. $32,2 \%$ ), hatten häufiger die Pflegestufe 3 oder mehr (55 vs. 11,2\%), waren häufiger desorientiert (40 vs. $20,4 \%$ ), inkontinent (70 vs. $26,9 \%$ ), bettlägerig ( 20 vs. $5,8 \%$ ) oder rollstuhlpflichtig (55 vs. $15,5 \%$ ); sie hatten häufiger einen Dekubitus (10 vs. 2,6\%) und einen Harnwegskatheter (55 vs. 6,4\%). Drei Viertel der Patienten des Intensivpflegedienstes hatten ein Tracheostoma, $70 \%$ waren aktuell beatmet; dies traf auf keinen der Patienten der anderen Pflegedienste zu. Patienten des Intensivpflegedienstes hatten ebenfalls häufiger positive Anamnesen für Krankenhausaufenthalte, Antibiotikagaben oder Operationen in der Vorgeschichte und wiesen am Erhebungstag häufiger eine Antibiotikatherapie oder eine Infektion auf.

Bei 3,7\% der untersuchten Patienten wurde MRSA, bei 14,4\% ESBL/MRGN gefunden, darunter bei $7,6 \%$ der Patienten einschließlich einer Resistenz 
Bundesgesundheitsbl 2016 ·59:292-299 DOI 10.1007/s00103-015-2290-7

(c) Springer-Verlag Berlin Heidelberg 2015

\section{N. Neumann - D. Mischler · C. Cuny · M. Hogardt · V.A.J. Kempf · U. Heudorf}

Multiresistente Erreger bei Patienten ambulanter Pflegedienste
im Rhein-Main-Gebiet 2014. Prävalenz und Risikofaktoren

\section{Zusammenfassung}

Hintergrund. Aus der ambulanten

Pflege gibt es bislang keine Unter-

suchung zur Prävalenz multiresistenter gramnegativer Erreger (MRGN) bzw. von Enterobakterien mit erweiterter Resistenz gegen $\beta$-Laktamantibiotika (ESBL) und nur wenige Daten zur Prävalenz von methicillinresistenten Staphylococcus aureus (MRSA). Deswegen führte das MRE-Netz Rhein-Main eine Untersuchung zur aktuellen Prävalenz und zu Risikofaktoren für MRGNKolonisationen/-Infektionen durch.

Material und Methoden. Charakteristika aller Patienten wurden mit einem modifizierten Fragebogen des europaweiten HALT-Projekts (healthcare associated infections in long term care facilities) erhoben und Nasen- und Rachenabstriche auf MRSA sowie Analabstriche auf ESBL/3MRGN/ 4MRGN untersucht. Risikofaktoren für eine MRE-Besiedelung wurden mittels Odds Ratio errechnet.

Ergebnisse. Zehn Pflegedienste mit insgesamt 486 Patienten nahmen teil, darunter ein Intensivpflegedienst mit beatmeten Patienten. Nasen-, Rachenabstriche ließen sich 269 Patienten entnehmen und 132 Patienten stimmten einem Analabstrich zu. Bei $3,7 \%$ wurden MRSA und bei $14,4 \%$ ESBL/ MRGN nachgewiesen $(6,8 \%$ ESBL, 7,6\% $3 \mathrm{MRGN}, 0 \%$ 4MRGN). Als Risikofaktoren für eine Besiedelung mit MRSA wurden Pflegestufe 3 oder mehr (OR 5,1), Antibiotikagabe innerhalb der letzten drei Monate (OR 3,7), Krankenhausaufenthalte innerhalb der letzten sechs Monate $(\mathrm{OR} 4,3)$ und MRSA in der Anamnese (OR 18,1) bestätigt. Inkontinenz sowie Krankenhausaufenthalt in der Anamnese wurden als Risikofaktoren für eine ESBL-Besiedelung gefunden (OR 9,5 resp. 6,5). Als Risikofaktoren für eine Besiedelung mit 3MRGN stellten sich eine hohe Pflegestufe $(O R 7,5)$ sowie Harnwegskatheter (OR 8,3), PEG und andere Stomata (OR 6,2) und invasive Beatmung (OR 5) sowie eine positive MRE-Anamnese (MRSA OR 20; ESBL OR 6,7) heraus.
Schlussfolgerung. Angesichts der hohen Prävalenzen an MRSA und 3MRGN müssen ambulante Pflegedienste über Kompetenz in der Pflege von Menschen mit MRE verfügen: gute Hygienemaßnahmen einschließlich Händehygiene und sachgerechtem Umgang bei der Wundversorgung und bei Punktionen und Injektionen, beim Umgang mit Kathetern, Stomata und ggf. invasiver Beatmung. Die entsprechenden KRINKOEmpfehlungen sind auch in der ambulanten Pflege umzusetzen.

\section{Schlüsselwörter}

Multiresistente Erreger (MRE) .

Methicillinresistenter Staphylococcus aureus (MRSA) · Enterobakterien mit erweiterter Resistenz gegen $\beta$-Laktamantibiotika (ESBL) . Multiresistente gramnegative Bakterien (MRGN) · Ambulante Pflegedienste . MRE-Netz

\section{Multidrug-resistant organisms (MDRO) in patients in outpatient care in the Rhine-Main region, Germany, in 2014. Prevalence and risk factors}

\section{Abstract}

Background. Data on the prevalence of methicillin-resistant Staphylococcus aureus (MRSA) in outpatient care are scarce and those on the prevalence of multidrug-resistant Gram-negative bacteria (MRGN) are lacking completely. Therefore, the network on multidrug-resistant organisms (MDRO) in the Rhine-Main region (MRE-Netz Rhein-Main) performed a multicenter study on current prevalence data and risk factors for MDRO. Materials and methods. Characteristics of all patients were obtained according to a modified healthcare-associated infections in long-term care facilities (HALT) questionnaire and swabs from the nares/throat and anus were tested for MRSA and extended-spectrum beta-lactamase (ESBL)/MRGN. Risk factors were calculated via odds ratios.

Results. Ten nursing services with 486 patients participated in this study, 269 pa- tients agreed to having swabs of the nares/throat taken, and 132 patients had anal swabs. MRSA was detected in $3.7 \%$, and ESBL/MRGN in $14.4 \%$ of the patients $(6.8 \%$ ESBL, $7.6 \%$ MRGN, $0 \%$ MRGN). Risk factors for MRSA were high dependency on care (stage 3 or above; OR 5.1), antibiotic use during the preceding 3 months (OR 3.7), hospital stay during the last 6 months (OR 4.3), and a positive history for MRSA (OR 18.1). Incontinence and preceding hospital stays proved to be risk factors for ESBL colonization (OR 9.5 or 6.5 ), whereas risk factors for MRGN colonization were a high level of care dependency (OR 7.5), urinary catheter (OR 8.3), percutaneous endoscopic gastrostomy tube and other stomata (OR 6.2), and artificial respiration (OR 5), in addition to a positive history for MRSA (OR 20) and ESBL (OR 6.7).
Conclusion. Considering the high prevalence of colonization with MDRO in outpatient care, nursing services must be competent in caring for such patients: good hygiene procedures, including hand hygiene and appropriate handling in wound management, punctures and injections, with catheters, stomata, and if necessary with artificial respiration should be practiced. The guidelines of the German Commission on hospital hygiene and infection prevention should also be observed.

\section{Keywords}

Multidrug-resistant organisms (MDRO) . Methicillin-resistant Staphylococcus aureus (MRSA) - Multidrug-resistant Gram-negative bacteria (MRGN) - Extended-spectrum betalactamase producing enterobacteriaceae (ESBL) · Outpatient care $\cdot$ Nursing services gegen Ciprofloxacin, also 3MRGN nach KRINKO 2012). Patienten des Intensivpflegedienstes $(n=20)$ wiesen häufiger eine MRSA-Besiedelung auf als die
Patienten der anderen Pflegedienste (12,5 vs. 3,2\%); eine Besiedelung mit ESBL oder 3 MRGN lag bei $30 \%$ der Intensivpflegepatienten (alle $3 \mathrm{MRGN}$ ) im Vergleich zu
13,1 \% (7,4\% ESBL und 5,7 \% 3MRGN) der anderen Patienten vor.

Die MRSA-Stämme wurden im Nationalen Referenzzentrum für Sta- 
Tab. 1 Patienten der Pflegedienste: Patientencharakteristika, Risikofaktoren für multiresistente Erreger (MRE), anamnestische Angaben zu MRE, Krankenhausaufenthalten, Antibiotika und Operationen sowie Infektionen und Antibiotikatherapie und MRE-Nachweise am Tag der Untersuchung bei 486 Patienten (und differenziert nach Intensivpflegediensten und anderen Pflegediensten)

\begin{tabular}{|c|c|c|c|c|c|c|}
\hline & \multicolumn{2}{|c|}{$\begin{array}{l}\text { Alle Pflege- } \\
\text { dienste }\end{array}$} & \multicolumn{2}{|c|}{$\begin{array}{l}\text { Nur Pflegedienste } \\
\text { ohne Intensivpflege }\end{array}$} & \multicolumn{2}{|c|}{$\begin{array}{l}\text { Nur Intensiv- } \\
\text { pflegedienst }\end{array}$} \\
\hline Pflegedienste $(n)$ & 10 & & 9 & & 1 & \\
\hline Patienten $(n)$ & 486 & & 466 & & 20 & \\
\hline Patientencharakteristika & $n$ & $\%$ & $n$ & $\%$ & $n$ & $\%$ \\
\hline Alter $85 \mathrm{~J}$ und mehr ${ }^{\mathrm{a}}$ & 176 & 36,3 & 176 & 37,8 & 0 & 0 \\
\hline Geschlecht männlich & 160 & 32,9 & 150 & 32,2 & 10 & 50 \\
\hline Pflegestufe 3 und mehr ${ }^{a}$ & 63 & 12,9 & 52 & 11,2 & 11 & 55 \\
\hline Diabetes & 108 & 22,2 & 104 & 22,3 & 4 & 20 \\
\hline \multicolumn{7}{|c|}{ Körperliche Einschränkungen mit möglichen Hygieneproblemen } \\
\hline Desorientiertheit ${ }^{\mathrm{a}}$ & 103 & 21,2 & 95 & 20,4 & 8 & 40 \\
\hline $\begin{array}{l}\text { Inkontinenz (Harn-, und/oder Stuhl- } \\
\text { inkontinenz) }\end{array}$ & 139 & 39,5 & 125 & 26,9 & 14 & 70 \\
\hline Bettlägerigkeit $^{\mathrm{a}}$ & 31 & 6,4 & 27 & 5,8 & 4 & 20 \\
\hline Rollstuhlpflicht ${ }^{\mathrm{a}}$ & 83 & 17,1 & 72 & 15,5 & 11 & 55 \\
\hline \multicolumn{7}{|l|}{ Hautbarriereverletzungen } \\
\hline Dekubitus & 14 & 2,8 & 12 & 2,6 & 2 & 10 \\
\hline Andere Wunden & 38 & 7,8 & 37 & 7,9 & 1 & 5 \\
\hline \multicolumn{7}{|l|}{ Katheter und Stomata } \\
\hline Harnwegskatheter ${ }^{\mathrm{a}}$ & 41 & 8,4 & 30 & 6,4 & 11 & 55 \\
\hline Gefäßkatheter & 3 & 0,6 & 3 & 0,6 & 0 & 0 \\
\hline $\mathrm{PEG}^{\mathrm{a}}$ & 22 & 4,5 & 8 & 1,7 & 14 & 70 \\
\hline Tracheostoma $^{a}$ & 15 & 3,1 & 0 & 0 & 15 & 75 \\
\hline Urostoma $^{a}$ & 4 & 0,8 & 4 & 0,9 & 0 & 0 \\
\hline Kolostoma $^{a}$ & 8 & 1,6 & 7 & 1,5 & 1 & 5 \\
\hline Invasive Beatmung $^{\mathrm{a}}$ & 14 & 2,9 & 0 & 0 & 14 & 70 \\
\hline \multicolumn{7}{|l|}{ Anamnese } \\
\hline Antibiotika letzte drei Monate ${ }^{a}$ & 53 & 10,9 & 48 & 10,3 & 5 & 25 \\
\hline $\begin{array}{l}\text { Krankenhausaufenthalt letzte sechs } \\
\text { Monate }\end{array}$ & 110 & 22,6 & 102 & 21,9 & 8 & 40 \\
\hline Operation letzte 30 Tage $^{\mathrm{a}}$ & 12 & 2,5 & 10 & 2,1 & 2 & 10 \\
\hline \multicolumn{7}{|c|}{ Multiresistente Erreger - anamnestische Angaben } \\
\hline MRSA-Anamnese & 12 & 2,5 & 12 & 2,6 & 0 & 0 \\
\hline MRSA aktuell & 6 & 1,2 & 5 & 1,1 & 1 & 5 \\
\hline ESBL-/MRGN-Anamnese ${ }^{\mathrm{a}}$ & 10 & 2,1 & 7 & 1,5 & 3 & 15 \\
\hline ESBL/MRGN aktuell & 3 & 0,6 & 3 & 0,6 & 0 & 0 \\
\hline \multicolumn{7}{|c|}{ Antibiotikatherapie oder Infektion am Untersuchungstag } \\
\hline Antibiotikatherapie & 5 & 1 & 4 & 0,9 & 1 & 5 \\
\hline Infektion $^{\mathrm{a}}$ & 5 & 1 & 3 & 0,6 & 2 & 10 \\
\hline \multicolumn{7}{|l|}{ Multiresistente Erreger Befund } \\
\hline MRSA & $10 / 269$ & 3,7 & $8 / 253$ & 3,2 & $2 / 16$ & 12,5 \\
\hline ESBL und/oder 3MRGN ${ }^{\mathrm{a}}$ & $19 / 132$ & 14,4 & $16 / 122$ & 13,1 & $3 / 10$ & 30 \\
\hline ESBL & $9 / 132$ & 6,8 & $9 / 122$ & 7,4 & $0 / 10$ & 10 \\
\hline $3 \mathrm{MRGN}^{\mathrm{a}}$ & $10 / 132$ & 7,6 & $7 / 122$ & 5,7 & $3 / 10$ & 30 \\
\hline 4MRGN & $0 / 132$ & 0 & $0 / 122$ & 0 & $0 / 10$ & 0 \\
\hline
\end{tabular}

phylokokken typisiert: sieben Isolate ließen sich dem spa-Typ t003, dem Sequenztyp ST225 (Rhein-Hessen-Epi- demiestamm) zuordnen. Die restlichen drei Isolate waren mit den spa-Typen 6608 , t790 und t2122 dem klonalen Komplex
CC22 (Barnim-Epidemiestamm) zugeordnet.

Als signifikante Risikofaktoren für eine MRSA-Besiedelung (Tab. 2) konnten eine hohe Pflegebedürftigkeit (mindestens Pflegestufe 3; OR 5,1), eine positive Anamnese für MRSA (OR $18,1)$, Antibiotika in den letzten drei Monaten (OR 3,7) und ein Krankenhausaufenthalt in den letzten sechs Monaten $(\mathrm{OR} 4,4)$ gefunden werden. Auch bei Patienten mit Desorientiertheit, Inkontinenz, Bewegungseinschränkung sowie Hautbarriereverletzungen durch Wunden oder Stomata einschließlich Beatmung war das Risiko für eine Besiedelung mit MRSA erhöht (Odds Ratios >2), allerdings war dies bei der niedrigen Fallzahl nicht signifikant. In der logistischen Regression blieben eine Pflegestufe 3 und mehr sowie ein Krankenhausaufenthalt signifikant.

Als signifikante Risikofaktoren für eine ESBL-Besiedelung (• Tab. 2) wurden Inkontinenz (OR 9,6) und Krankenhausaufenthalt in den letzten sechs Monaten (OR 6,5) ermittelt. Desorientiertheit, Wunden und positive MRSA-Anamnese waren ebenfalls mit einem - allerdings nicht signifikant - höheren Risiko für eine ESBL-Besiedelung verbunden (OR $>2$ ). In der logistischen Regressionsanalyse erwies sich ein Krankenhausaufenthalt in der Anamnese weiterhin als signifikanter Risikofaktor, als zusätzlicher signifikanter Risikofaktor kam Desorientiertheit hinzu.

Eine Besiedelung mit 3MRGN war signifikant positiv assoziiert mit Katheter oder Stomata (Harnwegskatheter OR 6,7; Stomata/PEG OR 7,8; invasive Beatmung OR 10,1), Pflegestufe 3 und mehr (OR $5,6)$ und positiver Anamnese für MRSA (OR 17,1). Diese Faktoren blieben auch in der logistischen Regression signifikant. Erhöhte, aber nicht signifikante Odds Ratios (OR $>2$ ) wurden darüber hinaus bei Inkontinenz, Bettlägerigkeit und nach Krankenhausaufenthalt in den vorangegangenen letzten drei Monaten gesehen (- Tab. 2).

In - Tab. 3 ist der Vergleich der hier erhobenen Daten zu MRE bei Patienten ambulanter Pflegedienste mit den Ergebnissen anderer Pflegedienste in Deutschland sowie anderer nicht akutstationärer Einrichtungen im Rhein-Main-Gebiet zu- 


\section{Originalien und Übersichten}

Tab. 2 Patienten ambulanter Pflegedienste: Risikofaktoren bei Patienten mit und ohne MRE-Nachweis: Patientencharakteristika, Infektionen, Antibiotikatherapie und MRE-Anamnese

\begin{tabular}{|c|c|c|c|c|c|c|}
\hline & \multicolumn{2}{|l|}{ MRSA } & \multicolumn{2}{|l|}{ ESBL } & \multicolumn{2}{|l|}{ 3MRGN } \\
\hline & OR & OR P5-P95 & OR & OR P5-P95 & OR & OR P5-P95 \\
\hline Alter mindestens 85 Jahre & 0,236 & $0,029-1,891$ & 0,593 & $0,118-2,984$ & 0,889 & $0,218-3,621$ \\
\hline Geschlecht männlich & 0,497 & $0,140-1,764$ & 0,916 & $0,234-3,576$ & 0,732 & $0,202-2,661$ \\
\hline Pflegestufe 3 und mehr & 5,167 & $1,432-18,636$ & 0,886 & $0,175-4,496$ & 5,596 & $1,470-21,311$ \\
\hline Diabetes & 0,294 & $0,037-2,364$ & 0,748 & $0,148-3,781$ & 1,169 & $0,285-4,788$ \\
\hline \multicolumn{7}{|c|}{ Körperliche Einschränkungen mit Hygieneproblemen } \\
\hline Desorientiertheit & 2,164 & $0,591-7,919$ & 2,593 & $0,653-10,298$ & 1,329 & $0,323-5,462$ \\
\hline Inkontinenz (Harn u/o Stuhl) & 1,785 & $0,504-6,326$ & 9,571 & $1,162-78,863$ & 2,702 & $0,667-10,936$ \\
\hline Bettlägerigkeit & 1,328 & $0,160-11,015$ & 1,058 & $0,122-9,143$ & 2,312 & $0,440-12,162$ \\
\hline Rollstuhlpflichtig & 2,211 & $0,604-8,094$ & 1,077 & $0,256-4,532$ & 1,491 & $0,398-5,592$ \\
\hline \multicolumn{7}{|l|}{ Hautbarriereverletzungen } \\
\hline Dekubitus & 0,962 & $0,938-0,985$ & 2,95 & $0,307-28,363$ & 0,929 & $0,885-0,975$ \\
\hline Andere Wunde & 2,565 & $0,514-12,802$ & 3,619 & $0,653-20,044$ & 3,167 & $0,583-17,189$ \\
\hline \multicolumn{7}{|l|}{ Katheter und Stomata } \\
\hline Harnwegskatheter & 0,761 & $0,093-6,202$ & 0,644 & $0,076-5,435$ & 6,687 & $1,740-25,702$ \\
\hline Stoma/PEG, Darm Trachea & 2,34 & $0,471-11,630$ & 0,921 & $0,873-0,972$ & 7,786 & $2,001-30,297$ \\
\hline Invasive Beatmung & 2,767 & $0,319-24,003$ & 0,924 & $0,877-0,973$ & 10.114 & $1,998-51,199$ \\
\hline \multicolumn{7}{|c|}{ Anamnese Krankenhausaufenthalt, Antibiotika und Operationen } \\
\hline $\begin{array}{l}\text { Antibiotikagabe innerhalb der letzten drei } \\
\text { Monate }\end{array}$ & 3,761 & $1,015-13,940$ & 1,911 & $0,364-10,019$ & 1,672 & $0,326-8,585$ \\
\hline $\begin{array}{l}\text { Krankenhausaufenthalt innerhalb der letzten } \\
\text { sechs Monate }\end{array}$ & 4,386 & $1,201-16,025$ & 6,483 & $1,525-27,556$ & 3,100 & $0,840-11,445$ \\
\hline Operation innerhalb der letzten drei Monate & 3,486 & $0,393-30,920$ & 0,900 & $0,886-0,975$ & 15.125 & $1,877-121,854$ \\
\hline \multicolumn{7}{|l|}{ MRE-Anamnese } \\
\hline MRSA-Anamnese & 18,071 & $3,736-87,411$ & 2,95 & $0,307-28,363$ & 17.143 & $2,913-100,895$ \\
\hline MRSA aktuell anamnestisch & 110,571 & $10,188-1200,0$ & 7,563 & $0,618-92,575$ & 6,722 & $0,555-81,415$ \\
\hline ESBL-Anamnese & 0,9614 & $0,938-0,985$ & 0,928 & $0,884-0,974$ & 5,900 & $0,986-35,315$ \\
\hline ESBL aktuell & 0,9624 & $0,940-0,986$ & 0,931 & $0,889-0,976$ & 0,931 & $0,889-0,976$ \\
\hline \multicolumn{7}{|l|}{ Am Erhebungstag } \\
\hline Antibiotika am Erhebungstag & 0,962 & $0,940-0,986$ & 0,9308 & $0,888-0,975$ & 0,931 & $0,888-0,975$ \\
\hline Infektion am Erhebungstag & 0,962 & $0,940-0,986$ & 0,9308 & $0,888-0,975$ & 0,931 & $0,888-0,975$ \\
\hline
\end{tabular}

sammenfassend dargestellt. Die MRSAPrävalenz von $3,7 \%$ lag im gleichen Bereich wie bei einer im Jahr 2011/12 durchgeführten Untersuchung in Münster/ Westfalen $(3,4 \%)$ [8] und einer im Jahr 2003 durchgeführten Untersuchung in Frankfurt $(2,7 \%)$ [9], wohingegen eine in den Jahren 2009/10 durchgeführten Untersuchung von Patienten ambulanter Pflegedienste in Münster eine deutlich höhere MRSA-Prävalenz (12\%) [10] erbracht hatte. Da bislang keine Daten zur Prävalenz an ESBL oder MRGN bei Patienten ambulanter Pflegedienste in Deutschland publiziert sind, ist ein externer Vergleich der im Rahmen unserer Studie erhaltenen Daten mit anderen Pflegediensten nicht möglich.
Der Vergleich mit anderen Untersuchungen im Rhein-Main-Gebiet in den letzten Jahren zeigt, dass die MRSAPrävalenz der Patienten der ambulanten Pflegedienste mit 3,7\% deutlich unter der MRSA-Prävalenz bei Altenpflegeheimbewohnern (6,5\% resp. 9,2\%) [14, $15]$, aber über der MRSA-Prävalenz bei ambulanten Dialysepatienten $(2,1 \%)$ [16] und Rehabilitationspatienten $(0,7 \%$, resp. 1,8\%) [17, 18] liegt. Auch die ESBLPrävalenz der Patienten der ambulanten Pflegedienste liegt mit 14,4\% fast doppelt so hoch wie bei ambulanten Dialysepatienten $(7,5 \%)$ [16] und Rehabilitationspatienten $(7,7 \%$ resp. $8,9 \%)[17,18]$, aber deutlich unter den bei Altenpflegeheimbewohnern festgestellten Raten (17,8\% resp. $26,7 \%)[14,15]$.

\section{Diskussion}

Im Rahmen des demografischen Wandels werden immer mehr Menschen in Deutschland pflegebedürftig werden und die Zahl der Menschen in stationärer oder mit ambulanter Pflege wird zunehmen. Es wurde geschätzt, dass unter der Annahme einer gleich bleibenden Pflegebedürftigkeit in der Bevölkerung die Zahl der Pflegebedürftigen von 2,25 Mio. im Jahr 2007 auf 2,65 Mio. im Jahr 2015 zunehmen wird und im Jahr 2030 etwa 3,37 Mio. Pflegebedürftige zu erwarten sind. Der Anteil der Pflegebedürftigen an der Gesamtbevölkerung wird von 2,7 auf 4,4\% im Jahr 2030 steigen Selbst unter der Annahme, dass sich bei steigender Lebenserwartung die Pflegebedürftigkeit 
Tab. 3 Vergleich der MRE-Prävalenzen bei den Patienten der ambulanten Pflegedienste im Rhein-Main-Gebiet mit den Daten zu MRSA-

Prävalenzen in Pflegediensten in Deutschland und mit den Daten zur MRE-Prävalenz in anderen außer(akut)klinischen Einrichtungen im Rhein-

Main-Gebiet

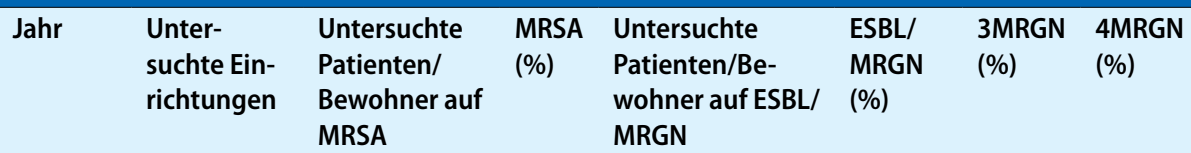

\begin{tabular}{|c|c|c|c|c|c|c|c|c|c|}
\hline \multicolumn{10}{|c|}{ Pflegedienste in Deutschland } \\
\hline Frankfurt/M & $2000 / 01$ & k.A. & 40 & 2,7 & n.u. & n.u. & n.u. & n.u. & Brune [9] \\
\hline Flensburg & $2009 / 10$ & k.A. & 41 & 12,0 & n.u. & n.u. & n.u. & n.u. & $\begin{array}{l}\text { Kreis Schleswig- } \\
\text { Flensburg [10] }\end{array}$ \\
\hline Münster Westfalen & $2011 / 12$ & 1 & 175 & 3,4 & n.u. & n.u. & n.u. & n.u. & Dornhöver et al. [8] \\
\hline Diese Untersuchung & 2014 & 10 & 269 & 3,7 & 132 & 14,4 & 7,6 & 0 & \\
\hline \multicolumn{10}{|c|}{ Untersuchungen im Rhein-Main-Gebiet } \\
\hline Dialysepatienten & 2012 & 15 & 751 & 2,1 & 532 & 7,5 & 3,8 & 0 & Dawson et al. [16] \\
\hline $\begin{array}{l}\text { Altenpflegeheim- } \\
\text { bewohner }\end{array}$ & 2012 & 8 & 184 & 9,2 & 150 & 26,7 & 21,4 & 0 & Heudorf et al. [14] \\
\hline $\begin{array}{l}\text { Altenpflegeheim- } \\
\text { bewohner }\end{array}$ & 2013 & 24 & 690 & 6,5 & 455 & 17,8 & 12,5 & 0 & Hogardt et al. [15] \\
\hline $\begin{array}{l}\text { Patienten aus Re- } \\
\text { habilitationskliniken }\end{array}$ & 2013 & 4 & 278 & 1,8 & 147 & 8,9 & 0,7 & 0 & Heudorf et al. [17] \\
\hline $\begin{array}{l}\text { Patienten aus Re- } \\
\text { habilitationskliniken }\end{array}$ & 2014 & 21 & 2440 & 0,7 & 1434 & 7,7 & 3,6 & 0,1 & Heudorf et al. [18] \\
\hline Diese Untersuchung & 2014 & 10 & 269 & 3,7 & 132 & 14,4 & 7,6 & 0 & \\
\hline
\end{tabular}

in ein höheres Alter verlagert (Szenario „sinkende Pflegequoten“), werden für das Jahr 2030 noch 3,3 Mio. Pflegebedürftige erwartet [19].

Im Jahr 2011 betrug die Zahl der Pflegebedürftigen in Deutschland insgesamt 2,5 Mio., von denen ca. 743.000 in Heimen und 1.758.000 zu Hause gepflegt wurden, 576.000 von ihnen zusammen mit oder ganz durch ambulante Pflegedienste [20]. Im Jahr 2011 gab es 12.300 zugelassene ambulante Pflegedienste, $63 \%$ davon in privater, $36 \%$ in freigemeinnütziger und $1 \%$ in öffentlicher Trägerschaft. Bezogen auf die Pflegebedürftigen wurden jeweils etwa die Hälfte von privaten und freigemeinnützigen Pflegediensten versorgt, weniger als $2 \%$ von Diensten in öffentlicher Trägerschaft. Im Mittel betreut ein Pflegedienst 46,7 Pflegebedürftige [21].

Zur Situation in der stationären Pflege wurden im Rahmen der europaweiten HALT-Studie (health-care associated infections in long-term care facilities) umfangreiche Daten zu den Bewohnercharakteristika (Alter, Geschlecht, Desorientiertheit, Inkontinenz, Bewegungseinschränkungen, Wunden, Versorgung mit Kathetern etc.) erhoben und publiziert $[11,12]$. Darüber hinaus liegen verschiedene Untersuchungen zur MREPrävalenz bei Altenpflegeheimbewohnern vor (Literatur in [22]) [14, 15]. Im Gegensatz dazu gibt es praktisch keine Erhebungen in der ambulanten Pflege zu den Patientencharakteristika und nur wenige Untersuchungen zur MRSAPrävalenz [8-10] sowie keinerlei Daten zur ESBL- oder MRGN-Prävalenz bei den durch ambulante Pflegedienste betreuten Pflegebedürftigen in Deutschland.

Vor diesem Hintergrund wurde die vorliegende Untersuchung durchgeführt. Leider waren nur 10 der 40 angefragten Pflegedienste zur Teilnahme bereit, denn dies bedeutete zunächst einen erheblichen Kommunikationsaufwand mit den Pflegebedürftigen oder ihren Betreuern im Hinblick auf die Information zum Sinn und Zweck der Untersuchung und die Einwilligung zur Teilnahme. Nur etwa die Hälfte der betreuten Pflegebedürftigen stimmte der Entnahme eines Nasen- und/oder Rachenabstrichs zur Untersuchung auf MRSA zu und etwa ein Viertel der Patienten war mit der Entnahme eines tiefen Analabstrichs und der Untersuchung auf ESBL/MRGN einverstanden. Mangels geeigneter Vergleichsdaten kann keine Aussage zur Repräsentativität der Pflegedienste und der
Pflegebedürftigen gemacht werden. Es handelte sich um einen Intensivpflegedienst, der zahlreiche beatmete Patienten versorgt, und neun „normale“ Pflegedienste. Von den hier versorgten Pflegebedürftigen waren $23 \%$ in Pflegestufe 0 , $42 \%$ in Pflegestufe 1, 22\% in Pflegestufe 2 und $13 \%$ in Pflegestufe 3 und höher eingestuft. Bundesweit betrug der Anteil der durch ambulante Pflegedienste versorgten Menschen $56 \%$ bei Pflegestufe 1, $33 \%$ bei Pflegestufe 2 und $11 \%$ bei Pflegestufe 3, wobei in Hessen der Anteil der Patienten mit Pflegestufe 3 und mehr bei $13 \%$ lag [21] und somit dieser Anteil weitgehend mit den in unserer Untersuchung erhobenen Daten übereinstimmt.

Im Vergleich mit der Erhebung der Bewohnercharakteristika von $6496 \mathrm{Be}$ wohnern in 70 Altenpflegeheimen in Deutschland [23] und einer Vollerhebung bei 3732 Bewohnern in allen 40 Altenpflegeheimen in Frankfurt am Main [24] in den Jahren 2010 und 2011 lag der Anteil der über 85-Jährigen bei den durch die ambulanten Pflegedienste betreuten Pflegebedürftigen deutlich niedriger (36\% im Vergleich zu 48-61\%); dies betraf auch den Anteil der Patienten mit Desorientiertheit (21\% im Vergleich zu 57-59\%), Inkontinenz (40\% im Ver- 
gleich zu 75-80\%) und Bewegungseinschränkung (24\% im Vergleich zu 45$51 \%)$. Demgegenüber wurden bei Harnwegskathetern ( 8,4 vs. $10 \%)$, Gefäßkathetern (0,6 vs. $0,2-0,3 \%)$, Dekubitus und Wunden (10,6 vs. $9 \%$ ) vergleichbare Prävalenzen gefunden. Nach Krankenhausaufenthalt in den letzten sechs Monaten oder einer Antibiotikatherapie in den letzten drei Monaten war im Rahmen der HALT-Studien in der stationären Altenpflege nicht gefragt worden, jedoch nach Operationen in den letzten 30 Tagen. Dort hatten 1,3-1,8\% der Bewohner eine Operation in dem Monat vor der Untersuchung angegeben [23, 24], bei den Pflegebedürftigen der ambulanten Pflegedienste lag der Anteil mit 2,5\% in einem vergleichbaren Bereich.

Bislang liegen nur sehr spärliche Erkenntnisse zur Prävalenz von Patienten mit MRSA/MRE im ambulanten pflegerischen Bereich in Deutschland vor. Bei Befragungen in Essen waren bis $1 \%$ der zu Pflegenden bekannte MRSATräger [25], bei Befragungen in Mittelhessen lag deren Rate bei 1,7\% [26]. Untersuchungen beispielsweise in Altenpflegeheimen haben gezeigt, dass nur ein geringer Teil der MRSA-Träger bekannt ist, die tatsächliche, bei Screeninguntersuchungen festzustellende Zahl liegt um ein Mehrfaches darüber [14]. So überrascht es nicht, dass bei den wenigen vorliegenden mikrobiologischen Untersuchungen bei von ambulanten Pflegediensten Betreuten die MRSA-Raten bei 3-12\% lagen [8-10]. Die in unserer Untersuchung gefundene Prävalenz von 3,7\% MRSA-besiedelter Pflegebedürftiger liegt somit im erwarteten Bereich.

Auch die erhaltenen spa-Typen mit $70 \%$ Anteil an 003 stimmten mit anderen bisherigen Untersuchungen des Netzwerks im Rhein-Main-Gebiet überein. Hinweise auf Transmissionen durch das Pflegepersonal ergaben sich nicht. Alle gefundenen MRSA spa-Typen entsprechen den klassischen krankenhausassoziierten MRSA, life-stock assoziierte LA-MRSA bzw. community-associated CA-MRSA wurden nicht festgestellt. In einer größeren Untersuchung bei über 2000 Rehabilitationspatienten in der Region wurde bei über $60 \%$ der Isolate der spa-Typ t003, bei $15 \%$ der Isolate der
spa-Typ t 032 und bei jeweils einem Isolat die spa-Typen t 034, t 127 und 608 nachgewiesen [18].

Im Vergleich mit aktuellen Untersuchungen zur MRSA-Prävalenz im außer(akut)klinischen Bereich waren die Patienten der ambulanten Pflegedienste sehr viel seltener als Bewohner von Altenpflegeheimen (aber häufiger als Patienten der ambulanten Dialyse und Rehabilitationspatienten) mit MRSA besiedelt. Die bekannten Risikofaktoren für eine MRSA-Besiedelung [22], nämlich eine positive MRSA-Anamnese, Krankenhausaufenthalt in der Anamnese, Hautbarriereverletzungen und Katheter, konnten in unserer Untersuchung bestätigt werden, darüber hinaus stellten sich eine Antibiotikabehandlung in den vorangegangenen drei Monaten und eine hohe Pflegestufe als signifikante Risikofaktoren für eine MRSA-Besiedelung heraus.

Da Daten zur ESBL- und MRGNPrävalenz bei Patienten ambulanter Pflegedienste in Deutschland bislang nicht publiziert sind, können hier keine Vergleiche angestellt werden. Die Besiedelungsraten der Pflegebedürftigen mit ESBL und MRGN lagen etwa doppelt so hoch wie bei Patienten der ambulanten Dialyse und bei Rehabilitationspatienten, aber deutlich niedriger als bei Altenpflegeheimbewohnern im Rhein-MainGebiet. Inkontinenz und Krankenhausaufenthalt in der Anamnese stellten sich als signifikante Risikofaktoren für eine ESBL-Besiedelung heraus. Im Hinblick auf eine MRGN-Besiedelung wurden eine positive Anamnese für MRSA, ein hohe Pflegestufe, Katheter, Stomata und Beatmung als signifikante Risikofaktoren gefunden, während ein Krankenhausaufenthalt in der Anamnese knapp die Signifikanz verfehlte.

Die Prävalenz einer Besiedelung mit ESBL lag bei den Pflegebedürftigen nur knapp über der ESBL-Prävalenz in der Allgemeinbevölkerung, die Prävalenz einer Besiedelung mit 3MRGN war jedoch etwa dreifach so hoch wie in der Allgemeinbevölkerung. Bislang wurden häufig Auslandsreisen und Krankenhausaufenthalte im Ausland - insbesondere in Ländern des Nahen und Fernen Ostens, aber auch in Südosteuropa als Risiko- faktoren für eine ESBL- oder MRGNBesiedelung publiziert [4-6]. In unserer Untersuchung konnte dies nicht nachvollzogen werden, da nur ein Patient über einen Auslandsaufenthalt in den vorangegangenen sechs Monaten - und dies ohne Krankenhausaufenthalt - berichtet hatte. Inkontinenz war mit einem hohen Risiko für ESBL-Besiedelung assoziiert. Grundsätzlich kann damit auch das Risiko für eine Harnwegsinfektion durch ESBL verbunden sein, auch wenn dies in unserer Studie mit einer relativ kleinen Probandenzahl so nicht nachgewiesen werden konnte. Jedoch stellten sich Katheter oder Stomata sowie Kontakte zum Medizinsystem (Krankenhausaufenthalte und Operationen) als Risikofaktoren für eine Besiedelung mit 3MRGN heraus. Diesem Befund sollte durch weitere Untersuchungen nachgegangen werden. Bei Bestätigung sollte dies bei Empfehlungen zum Screening auf ESBL/MRGN in medizinischen Einrichtungen berücksichtigt werden.

Unsere Untersuchung unterstreicht, dass sich ambulante Pflegedienste auf die Versorgung von Menschen mit MRE einstellen und über Kompetenz in der Pflege von Menschen mit MRE verfügen müssen. Dazu gehört eine gute Händehygiene und sachgerechter Umgang bei der Wundversorgung und bei Punktionen und Injektionen sowie beim Umgang mit Kathetern, Stomata und ggf. invasiver Beatmung. Darüber hinaus ist die Kenntnis der Übertragungswege von MRSA und ESBL/MRGN unabdingbar, um die erforderlichen zusätzlichen Hygienemaßnahmen zu ergreifen. Die entsprechenden KRINKO-Empfehlungen [27] sind auch in der ambulanten Pflege umzusetzen. Die MRE-Netzwerke sind aufgerufen, die ambulanten Pflegedienste hierbei durch Informationen und Fortbildungen zu unterstützen.

\section{Korrespondenzadresse}

\section{Prof. Dr. U. Heudorf}

Gesundheitsamt Frankfurt, MRE-Netz Rhein-Main

Breite Gasse 28, 60313 Frankfurt am Main ursel.heudorf@stadt-frankfurt.de 
Danksagung. Wir danken den teilnehmenden Pflegediensten und ihren Patienten für ihre Teilnahme. Unser Dank gilt auch dem Bundesministerium für Gesundheit für die Finanzierung des Projekts (ZMVI5-2514_NIK_003).

\section{Einhaltung ethischer Richtlinien}

Interessenkonflikt. N. Neumann, D. Mischler, C. Cuny, M. Hogarth, V.A.J. Kempf und U. Heudorf geben an, dass kein Interessenkonflikt vorliegt.

Alle im vorliegenden Manuskript beschriebenen Untersuchungen am Menschen wurden mit Zustimmung der zuständigen Ethik-Kommission, im Einklang mit nationalem Recht sowie gemäß der Deklaration von Helsinki von 1975 (in der aktuellen, überarbeiteten Fassung) durchgeführt. Von allen beteiligen Patienten liegt eine Einverständniserklärung vor.

Die Untersuchung ist durch die Ethikkommission der Landesärztekammer Hessen genehmigt worden (FF32/2014).

\section{Literatur}

1. The European Centre for Disease Prevention and Control (ECDC) Antimicrobial resistance surveillance in Europe 2013. ECDC 17. November 2014. http://www.ecdc.europa.eu/en/ publications/_layouts/forms/Publication DispForm.aspx?List=4f55ad51-4aed-4d32-b960af70113dbb90\&ID $=1205$

2. Gagliotti C, Balode A, Baquero F, Degener J, Grundmann H, Gür D, Jarlier V, Kahlmeter G, Monen J, Monnet DL, Rossolini GM, Suetens C, Weist K Heuer O, EARS-Net Participants (Disease Specific Contact Points for AMR) (2011) Escherichia coli and Staphylococcus aureus: bad news and good news from the European Antimicrobial Resistance Surveillance Network (EARS-Net, formerly EARSS), 2002 to 2009. Euro Surveill 16(11). pii:19819

3. Köck R, Werner P, Friedrich A et al (2012) Characteristics of Staphylococcus aureus nasal carriage, resistance patterns and genetic lineages in healthy German adults. In: 52nd interscience conference on antimicrobial agents and chemotherapy, San Francisco, USA

4. Tängdén T, Cars O, Melhus A, Löwdin E (2010) Foreign travel is a major risk factor for colonization with Escherichia coli producing CTX-Mtype extended-spectrum beta-lactamases: a prospective study with Swedish volunteers. Antimicrob Agents Chemother 54(9):3564-3568 doi:10.1128/AAC.00220-10

5. Lübbert C, Straube L, Stein C, Makarewicz O, Schubert S, Mössner J, Pletz MW, Rodloff AC (2015) Colonization with extended-spectrum beta-lactamase-producing and carbapenemaseproducing Enterobacteriaceae in international travelers returning to Germany. Int J Med Microbiol 305(1):148-156. doi:10.1016/j. ijmm.2014.12.001
6. Kuenzli E, Jaeger VK, Frei R, Neumayr A, DeCrom S, Haller S, Blum J, Widmer AF, Furrer H, Battegay M, Endimiani A, Hatz C (2014) High colonization rates of extended-spectrum $\beta$-lactamase (ESBL)producing Escherichia coli in Swiss travellers to South Asia- a prospective observational multicentre cohort study looking at epidemiology, microbiology and risk factors. BMC Infect Dis 14:528. doi:10.1186/1471-2334-14-528

7. Valenza G, Nickel S, Pfeifer Y et al (2014) Extendedspectrum- $\beta$-lactamase-producing Escherichia coli as intestinal colonizers in the German community. Antimicrob Agents Chemother 58:1228-1230. doi:10.1128/AAC.01993-13

8. Domhöver A, Köck R, Becker K, Mellmann A, Friedrich AW (2014) Prävalenz von Methicillinresistenten Staphylococcus aureus (MRSA) und mit MRSA-assoziierten Risikofaktoren in der ambulanten. Pflege Umweltmedizin Hygiene Arbeitsmedizin 19:426-430

9. Brune IR (2005) Prävalenz des Methicillinresistenten Staphylococcus aureus (MRSA) in ambulanter Pflege, Altenheim und Geriatrie in Frankfurt am Main 2000/2001. Goethe-Universität Frankfurt am Main; Dissertation

10. Schleswig-Flensburg K (2010) Projektbericht über ein regionales Netzwerk zur Intensivierung von Präventionsstrategien bei MRSA im Kreis Schleswig-Flensburg. http://sh-mre.de/files/uploads/17/1301474629_projektbericht.pdf. Zugegriffen: 15. Juli. 2015

11. Latour $\mathrm{K}$, Jans $B$, the HALT management team (2009) Healthcare associated infections in long-term care facilities. Results of the pilot point prevalence survey. Deposit number D/2011/2505/8; IPH/Epi-report number: 2011006. http://halt.wiv-isp.be/report/Reports/HALT-1/ HALT\%20Report\%20Pilot\%20Survey\%20Nov\%20 2009.pdf. Zugegriffen: 15. Juli. 2015

12. Ruscher C, Kraus-Haas M, Nassauer A, Mielke M (2015) Healthcare associated infections and antimicrobial use in long-term care facilities (HALT-2) Deutsche Ergebnisse der zweiten europäischen Prävalenzerhebung. Bundesgesundheitsbl Gesundheitsforsch Gesundheitsschutz 58:436-451

13. KRINKO Kommission für Krankenhaushygiene und Infektionsprävention (2012) Hygienemaßnahmen bei Infektion oder Besiedelung mit multiresistenten gramnegativen Stäbchen. Bundesgesundheitsbl Gesundheitsforsch Gesundheitsschutz 55:1311-1354

14. Heudorf U, Gustav C, Mischler D, Schulze J (2014) Nosokomiale Infektionen, systemischer Antibiotikaeinsatz und multiresistente $\mathrm{Er}$ reger bei Bewohnern von Altenpflegeheimen - das Frankfurter HALT plus MRE-Projekt, 2012. Bundesgesundheitsbl Gesundheitsforsch Gesundheitsschutz 57:414-422

15. Hogardt M, Proba P, Mischler D, Cuny C, Kempf VA, Heudorf U (2015) Current prevalence of multidrugresistant organisms in long-term care facilities in the Rhine-Main district, Germany, 2013. Eurosurveillance 20(26): pii =21171. www.eurosurveillance.org/ViewArticle.aspx\Articled $=21171$

16. Dawson A, Mischler D, Petit C, Klein R, Heudorf U, Herrmann M (2012) Prevalence of Methicillinresistant Staphylococcus aureus in end stage renal failure patients in Saarland and Hessen. Int J Med Microbiol 302:87
17. Heudorf U, Färber A, Nagel A, Kempf VAH, Mischler D (2014) Multiresistente Erreger in der Rehabilitation - Ergebnisse einer Pilotstudie des MRE-Netz Rhein-Main, 2013. Umweltmedizin Hygiene Arbeitsmedizin 19:410-417

18. Heudorf U, Färber D, Mischler D, Schade M, Zinn C, Cuny C, Nilius D, Herrmann M (2014) Multiresistente Erreger in Rehabilitationseinrichtungen im Rhein-Main-Gebiet, Deutschland. I. Prävalenz und Risikofaktoren. Rehabilitation 54:339-345

19. Statistische Ämter des Bundes und der Länder (2010) Demographischer Wandel in Deutschland. 2: Auswirkungen auf Krankenhausbehandlungen und Pflegebedürftige in Bund und Ländern, 2010

20. Internet DS. https://www.destatis.de/DE/ ZahlenFakten/GesellschaftStaat/Gesundheit/ Gesundheit.html;jsessionid=38C721D3F7F1AB7 B20ACA7CE6245FECF.cae1. Zugegriffen: 16. Juli. 2015

21. Statistisches Bundesamt: Pflegestatistik 2011: Pflege im Rahmen der Pflegeversicherung. Ländervergleich - Ambulante Pflegedienste 2013. https://www.destatis.de/DE/ZahlenFakten/ GesellschaftStaat/Gesundheit/Pflege/Pflege.html. Zugegriffen: 16. Juli. 2015

22. KRINKO Kommission für Krankenhaushygiene und Infektionsprävention (KRINKO) am Robert Koch-Institut (RKI) (2014) Empfehlungen zur Prävention und Kontrolle von Methicillin-resistenten Staphylococcus aureus-Stämmen (MRSA) in medizinischen und pflegerischen Einrichtungen. Bundesgesundheitsbl Gesundheitsforsch Gesundheitsschutz 57:696-732

23. Wischnewski N, Mielke M, Wendt C (2011) Healthcare-associated infections in long-term care facilities (HALT) Ergebnisse aus Deutschland im Rahmen einer europäischen Prävalenzstudie. Bundesgesundheitsbl Gesundheitsforschung Gesundheitsschutz 54:1147-1152. doi:10.1007/ s00103-011-1363-5

24. Heudorf U, Boehlcke K, Schade M (2012) Healthcare-associated infections in long-term care facilities (HALT) in Frankfurt am Main, Germany, January to March 2011. Eurosurveillance 17: 35ff

25. Popp W, Ross B, Raffenberg M, Sanewski A, Scheytt C, Schwermer L et al (2012) Berichte aus den MRSA-Netzwerken - Sektorenübergreifende MRSA-Eintagesprävalenz - Erfahrungen aus Essen. Epidemiologisches Bulletin 27:250-254

26. Loss R, Ballmann G, Breitbach B, Haberer W, Harpel S, Hoffmann C et al (2012) Aufbau eines MRENetzwerks Mittelhessen. Erste Ergebnisse. Hyg Med 37:12-13

27. KRINKO Kommission für Krankenhaushygiene und Infektionsprävention. Empfehlungen der Kommission für Krankenhaushygiene und Infektionsprävention (KRINKO). http://www.rki. de/DE/Content/Infekt/Krankenhaushygiene/ Kommission/kommission_node.html;jsessionid=A 68919700509D9E1F4C79FD4F0A3EB22.2_cid363. Zugegriffen: 16. Juli. 2015 


\section{Hier steht eine Anzeige.}

Springer 